\title{
Chapter 21 \\ Prediction of LEAP-UCD-2017 Centrifuge Test Results Using Two Advanced Plasticity Sand Models
}

\author{
Long Chen, Alborz Ghofrani, and Pedro Arduino
}

\begin{abstract}
In accordance with the Liquefaction Experiments and Analysis Projects (LEAP)-UCD-2017 guidelines, two stress-dependent bounding surface constitutive models, Manzari-Dafalias and PM4Sand, were calibrated for Class-B prediction of centrifuge experiments of a sloped ground surface model of uniformly deposited Ottawa F-65 sand. Different calibration techniques and objectives were chosen for each material model. It was shown that both models were capable of simulating the behavior of cohesionless soils under liquefaction.
\end{abstract}

\subsection{Introduction}

Soil liquefaction induced by earthquakes can cause significant damage to adjacent structures and lead to considerable economic loss. The mechanism and effects of soil liquefaction have been studied extensively throughout the years. With the development of computational tools and advanced constitutive models which can capture complex soil behavior under various loading and drainage conditions, numerical modeling has become popular for predicting liquefaction-induced ground failure and deformations. For a numerical model to produce reasonable results, a comprehensive verification and validation study is necessary. Centrifuge tests, which can physically represent field conditions under earthquakes, have been used to provide benchmark studies for numerical analysis. VELACS (Verification of Liquefaction Analysis by Centrifuge Studies) (Arulanandan and Scott 1993) was a valuable initial attempt that identified the limitations of prevailing procedures. LEAP (Liquefaction Experiments and Analysis Projects) (Manzari et al. 2018) is a new effort to validate numerical models using centrifuge tests. In this work comparisons between experimental results and numerical

\footnotetext{
L. Chen · A. Ghofrani · P. Arduino ( $₫)$

Department of Civil and Environmental Engineering, University of Washington, Seattle, WA, USA

e-mail: longchen@uw.edu; alborzgh@uw.edu; parduino@uw.edu
} 
simulations obtained using two different constitutive models, i.e., Manzari-Dafalias (Dafalias and Manzari 2004) and PM4Sand (Boulanger and Ziotopolou 2015), implemented in the OpenSees finite element framework are presented and discussed.

\subsection{Backgrounds}

LEAP embodies a series of projects that attempt to extend understanding of the behavior of saturated granular soils subjected to seismic loading and at the same time provide high-quality experimental data sets for evaluation of constitutive models capable of reproducing liquefaction. Collectively these projects shed light on the performance of computational material models and numerical frameworks using benchmark centrifuge experiments representing different geotechnical settings. After an initial set of experiments in 2015 that resulted in the LEAP-GWU-2015 (Kutter et al. 2018) workshop, a second set of experiments was conducted at nine centrifuge facilities around the world, namely, Cambridge University (CU), Ehime University (Ehime), Korea Advanced Institute of Science and Technology (KAIST), Kyoto University (KyU), Taiwan National Central University (NCU), the University of California Davis (UCD), Rensselaer Polytechnic Institute (RPI), French Institute of Science \& Technology for Transportation, development and networks (IFSTTAR), and Zhejiang University (ZJU). Blind simulations were completed and submitted to the LEAP organizers, and a second workshop (LEAP-UCD2017) was organized at Davis at the end of 2017.

In all these studies, the well-characterized Ottawa F-65 sand (Bastidas 2016; Vasko 2015; El Ghoraiby et al. 2017, 2019) was used. During the second set of experiments, the sand was deposited uniformly in an instrumented 5-degree slope with a target density of $1652 \mathrm{~kg} / \mathrm{m}^{3}$. All centrifuge experiments used a rigid box container to eliminate complexities in boundary conditions. With small variations, depending on the configuration of each centrifuge facility, all models were prepared to represent a unique prototype size: $20 \mathrm{~m}$ in length, $4 \mathrm{~m}$ in height at the midpoint, and width greater than $9 \mathrm{~m}$ to reduce the effects of frictional boundary conditions on the container sidewalls. Four accelerometers (AH1-AH4) and four pore pressure transducers (P1-P4) were positioned along the centerline. A series of four $1 \mathrm{~Hz}$ ramped sine motions were imposed on each model. Motions 2 and 3 with a $0.15 \mathrm{~g}$ and $0.25 \mathrm{~g} \mathrm{PGA}$, respectively, were chosen strong enough to represent a destructive event. Motion 2 acceleration time history is shown in Fig. 21.1 and was used in all the Type B predictions presented in this paper. 
Fig. 21.1 Target input motion 2 acceleration time history
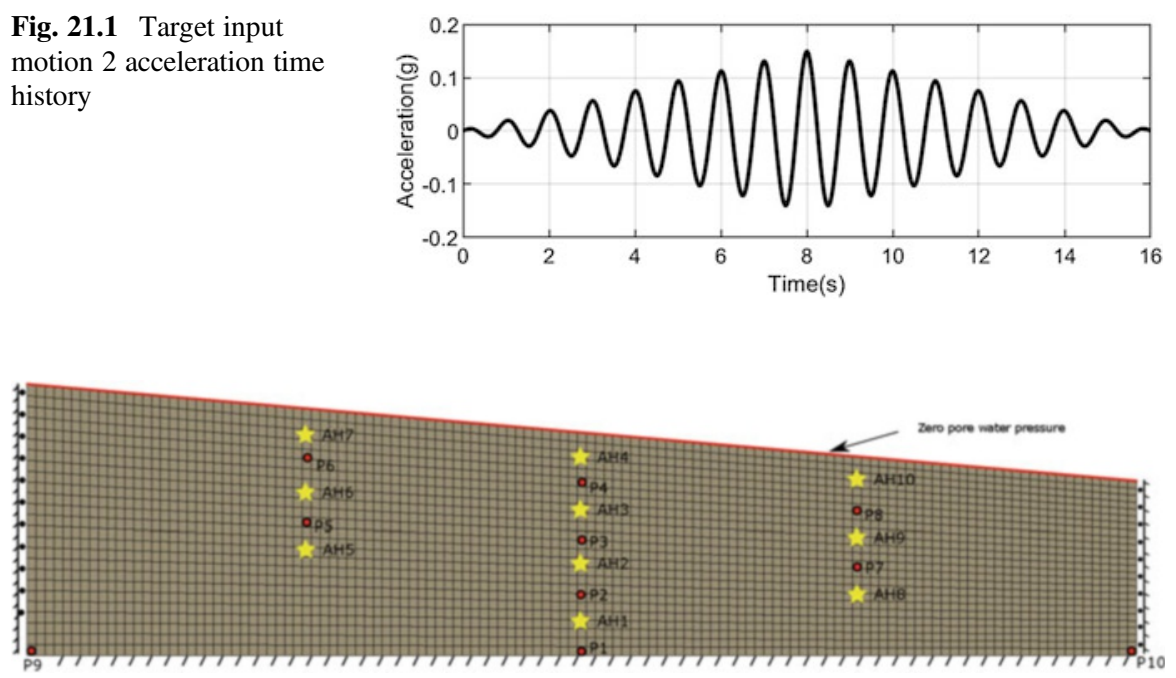

Fig. 21.2 Finite element mesh and location of recorded pore water pressures $(\mathrm{P})$ and accelerations $(\mathrm{AH})$

\subsection{FE Model Development}

In this work a 2D plane-strain representation of the problem was adopted, and numerical models were built in prototype scale using the OpenSees computational framework (McKenna 1997; OpenSees 2007). OpenSees (Open System for Earthquake Engineering Simulations) is an open-source, object-oriented finite element platform created and maintained by the Pacific Earthquake Engineering Research (PEER). The models consisted of 3125 four-node quadrilateral elements with an average size of $0.16 \mathrm{~m} \times 0.16 \mathrm{~m}$. The physically stabilized single-point integration and mixed displacement-pressure (u-p) element (SSPquadUP; see McGann et al. 2015) were used to capture the effective stress response of each simulated centrifuge test. A schematic of the mesh used in this work along with the location of recorded responses is depicted in Fig. 21.2. Appropriate mesh refinement was chosen to properly resolve the propagation of shear waves of up to $50 \mathrm{~Hz}$ in the soil domain.

\subsubsection{Boundary and Loading Conditions}

The bottom boundary was fixed against vertical movement. The acceleration time history was applied to both vertical boundaries and the base of the model using the so-called UniformExcitation loading pattern in OpenSees. This was done to account for a rigid container. Pore pressures at the slope surface nodes were set to be zero during the analysis to ensure drainage and avoid generation of excess pore pressures 

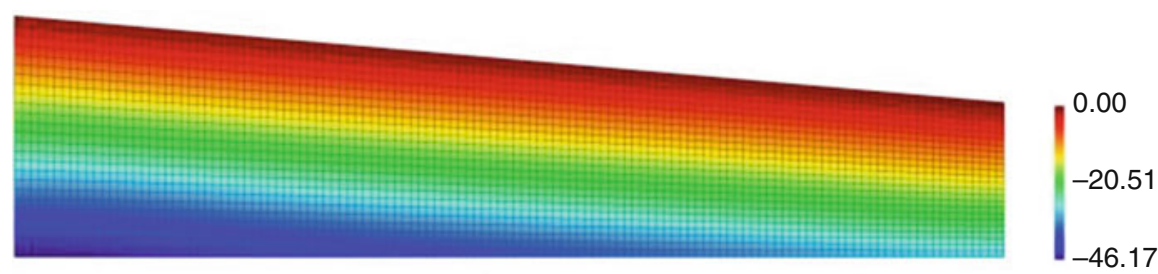

(c)

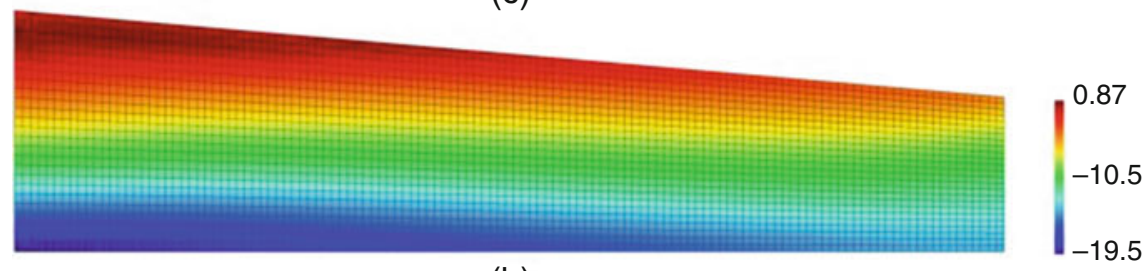

(b)

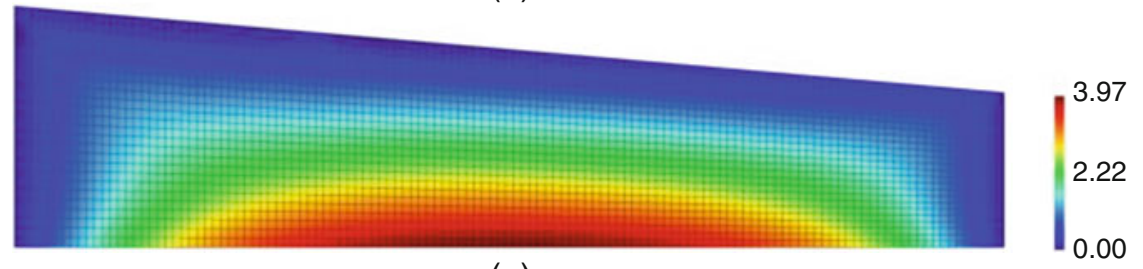

(a)

Fig. 21.3 Initial stress field after applying gravity. (a) Vertical effective stress distribution. (b) Horizontal effective stress distribution. (c) Shear stress distribution. All units in $\mathrm{kPa}$

at the surface. The free water on the slope was not modeled and effects of water sloshing (if any) were not considered. The soil was assumed to be always in contact with the container. A frequency-dependent Rayleigh damping was applied to compensate for small strain damping, which both MD and PM4Sand models lack in their formulation. The Rayleigh damping coefficients were chosen such that a $2 \%$ damping was obtained at $0.2 \mathrm{~Hz}$ and $20 \mathrm{~Hz}$. The permeability was adopted from Ghofrani and Arduino (2018) and set to be $3.0 \times 10 \mathrm{e}^{-5} \mathrm{~m} / \mathrm{s}$.

To apply gravity and centrifugal accelerations, the materials were set to be linear elastic, and elemental body force was increased gradually to reduce numerical instabilities in the model. Once gravity was in place, the materials were switched to have elastoplastic behavior, and enough extra steps were run to adjust their internal variables to any plastic behavior and maintain equilibrium. A Poisson's ratio of 0.3 was assumed to generate the initial stress state. This Poisson's ratio yielded a lateral earth pressure coefficient $K_{0}$ of 0.43 under level ground plane-strain conditions. Figure 21.3 shows the imposed initial state of stress in terms of vertical, horizontal, and shear stresses. After reaching the desired initial stress state, the acceleration time history was applied to the rigid boundaries. The constant average acceleration Newmark method $(\beta=0.25, \gamma=0.5)$ was used in order to resolve the integration in time. To account for the material nonlinearity, both models used explicit 
modified Euler integration schemes with sub-stepping error control (Sloan et al. 2001). After the main shake portion of the motion was over, additional dynamic analysis steps were executed to dissipate any excess pore pressure generated during the shaking phase.

\subsubsection{Material Constitutive Models}

The bounding surface constitutive models developed by Dafalias and Manzari (2004) (referred to as MD henceforth for brevity) and Boulanger and Ziotopolou (2015) (PM4Sand) were used for comparison purposes. The latter uses the basic framework of the model introduced by Dafalias and Manzari improved to better capture well-known trends in liquefiable soils. Both these models follow critical state soil mechanics concepts and are capable of capturing stress-strain relationships for denser-than-critical and looser-than-critical sands under different drainage and loading conditions. In the MD model, the relationship with critical state soil mechanics is through the so-called state parameter, defined as $\psi=e-e_{c}$ by Been and Jefferies (1985). Introducing a fabric tensor that evolves with accumulated plastic strain, MD can control contraction and dilation during cyclic loading.

Boulanger and Ziotopolou (2015) improved the capabilities of the MD model by developing a sand plasticity model for earthquake engineering applications that is simpler to calibrate and better represents some important aspects of liquefiable soils. Instead of the state parameter, a relative state parameter index, defined as $\xi_{R}=D_{R}$, cs $-D_{R}$ by Boulanger (2003), is adopted and used together with the empirical relationship for critical state line proposed by Bolton (1986). Although the number of model parameters for PM4Sand is larger than MD, the number of parameters that need to be calibrated is reduced with considerations added internally for the model to follow general soil behavior. By changing three primary input parameters, namely, shear modulus coefficient, $G_{0}$; apparent relative density, $D_{\mathrm{r}}$; and contraction rate parameter, $h_{\text {po }}$, the user can achieve reasonable approximations of desired behavior including pore pressure generation and dissipation, limiting strains, and cyclic mobility. Sending the "FirstCall" signal to the model allows users to initialize/reset secondary parameters (21 in total) and fabric history, while the "PostShake" signal allows the user to activate post-liquefaction functionality to improve simulation of reconsolidation strains. In the OpenSees analyses performed as part of this study, the "FirstCall" flag was called for material initialization just before switching to elastoplastic during gravity analysis. Obviously using the secondary parameters, the user can further fine-tune the response. Since its introduction, the PM4Sand model has drawn wide attention of geotechnical engineers and researchers due to its relatively easy calibration process and good agreement with field observations. More details on the model can be found at Boulanger and Ziotopolou (2015). 


\subsubsection{Calibration}

In order to predict the response of centrifuge tests, material parameters were calibrated using available laboratory test data. Several drained and undrained cyclic triaxial as well as cyclic simple shear tests were used for this purpose (El Ghoraiby et al. 2017, 2019). To calibrate the model parameters, the constitutive evolution equations were integrated independently of the FE framework using a constitutive driver (referred to as MixedDriver) implemented in $\mathrm{C}++$ based on the formulation proposed by Alawaji et al. (1992). The method uses an implicit backward Euler scheme to integrate the constitutive equations. Using this framework, it is possible to test any constitutive model under different stress paths and drainage conditions and independent of any finite element restriction. Also MixedDriver allows for simulating stress-controlled, strain-controlled, and mixed (stress-strain)-controlled loading conditions.

\section{Parameters}

Some of the MD constitutive model parameters can be estimated directly from drained and undrained monotonic triaxial tests. These parameters include $G_{0}, M c$, $c, n^{b}, n^{d}, \lambda_{c}, e 0$, and $\xi$. These parameters, in particular the last three, play an essential role in the response obtained from the MD model as the whole framework is built on the critical state surface concept. From the experimental standpoint, however, it is very difficult to obtain reliable data to represent the critical state of a soil. This has important implications in the MD model. Therefore, emphasis should be given to laboratory specimen sheared enough to reach critical state. The rest of the parameters can be calibrated using either trial and error or optimization techniques. Since the authors had experience working with Ottawa F65 sand from the previous LEAP 2015 exercise (Ghofrani and Arduino 2018), as well as few other projects, e.g., Ramirez et al. (2018), some of the same parameters used in previous calibration efforts were used for this study. Although it would have been ideal to calibrate the model for all of the characteristics revealed by the lab results at hand, due to intrinsic characteristics of the constitutive model, some specific objectives could not be achieved. In this context, the trend of number of cycles to reach liquefaction is one example. Based on experience, a number of cycles to liquefaction at smaller cyclic stress ratios do not play an important role in centrifuge experiments of this size and in particular are not observed with the loading conditions used in LEAP. Therefore, in this study the main objective when calibrating the MD model was to achieve a reasonable range of excess pore water pressure in cyclic tests as well as accumulation of shear strains, rather than trying to achieve perfect match between the number of cycles to reach liquefaction at different cyclic stress ratios. Table 21.1 summarizes the calibrated parameters for this phase of LEAP 2017. 
Table 21.1 Calibrated MD parameters and their values

\begin{tabular}{|c|c|c|}
\hline \multicolumn{2}{|l|}{ Parameter } & Value \\
\hline \multirow[t]{2}{*}{ Elastic } & $G_{0}$ & 82.35 \\
\hline & $v$ & 0.01 \\
\hline \multirow[t]{5}{*}{ Critical state } & $M$ & 1.35 \\
\hline & c & 0.70 \\
\hline & $\lambda c$ & 0.055 \\
\hline & $e_{0}$ & 0.80 \\
\hline & $\xi$ & 0.50 \\
\hline Yield surface & $m$ & 0.02 \\
\hline \multirow[t]{3}{*}{ Plastic modulus } & $h_{0}$ & 16.18 \\
\hline & $c_{\mathrm{h}}$ & 0.996 \\
\hline & $n^{\mathrm{b}}$ & 0.64 \\
\hline \multirow[t]{2}{*}{ Dilatancy } & $A_{0}$ & 0.75 \\
\hline & $n^{\mathrm{d}}$ & 1.50 \\
\hline \multirow[t]{2}{*}{ Fabric tensor } & $z_{\max }$ & 12.50 \\
\hline & $c_{\mathrm{z}}$ & 500.0 \\
\hline
\end{tabular}

\section{PM4Sand Parameters}

The biggest advantage in using PM4Sand is that the calibration process is relatively straightforward. As mentioned before, only 3 primary parameters and 4 secondary parameters (out of 21) were used for calibration in this study. The remaining secondary parameters were kept at their default values, previously calibrated by Boulanger and Ziotopolou (2015) to represent typical soil behavior.

Since PM4Sand was developed for plane-strain conditions, undrained cyclic plane-strain compression (PSC) conditions were used for calibration purposes. Initially three sets of parameters were calibrated for three void ratios, namely, $0.515\left(D_{\mathrm{r}}=90.5 \%\right), 0.542\left(D_{\mathrm{r}}=79.6 \%\right)$, and $0.585\left(D_{\mathrm{r}}=62.2 \%\right)$, in accordance with LEAP phase 1 guidance. Maximum and minimum void ratios for the Ottawa F65 sand were adopted from previous test data and used to calculate relative densities. The critical state effective friction angle $\phi_{\mathrm{cv}}$ was evaluated using shear and normal stresses at which the soil reached critical state during monotonic drained triaxial tests. The shear modulus coefficient $G_{0}$ was calibrated by matching the initial slope of the stress-strain curves in undrained cyclic tests. $H_{\mathrm{po}}$, which controls the rate of pore pressure generation between contraction and dilation, and $c_{\mathrm{z}}$, which controls the strain level at which fabric becomes relevant, were calibrated iteratively to match the experimental liquefaction strength curves. In contrast to the MD model, calibration of the PM4Sand model was done to capture the number of cycles to liquefaction observed in the triaxial tests. Table 21.2 presents the calibrated parameters for this phase of LEAP 2017.

Figure 21.4 depicts the case of a cyclic stress-controlled undrained triaxial test (El Ghoraiby et al. 2017, 2019) along with simulations obtained using MD and PM4Sand. The MD model was able to capture the asymmetry of the cyclic triaxial 
Table 21.2 Calibrated PM4Sand parameters and their values

\begin{tabular}{|c|c|c|c|c|}
\hline & & & $e_{o}=0.585$ & \\
\hline \multirow[t]{3}{*}{$\begin{array}{l}\text { Primary } \\
\text { parameters }\end{array}$} & $D_{\mathrm{r}}$ & Relative density & $62.2 \%$ & $\begin{array}{l}\text { Modified to } 65 \% \text { for Class-B } \\
\text { prediction }\end{array}$ \\
\hline & $G_{0}$ & Shear modulus coefficient & 350.0 & \\
\hline & $h_{\text {po }}$ & Contraction rate parameter & 0.07 & \\
\hline \multirow[t]{4}{*}{$\begin{array}{l}\text { Secondary } \\
\text { parameters }\end{array}$} & $e_{\max }$ & Maximum void ratio & 0.7389 & $\begin{array}{l}\text { Modified to match } 65 \% \text { rela- } \\
\text { tive density for Class-B } \\
\text { prediction }\end{array}$ \\
\hline & $e_{\min }$ & Minimum void ratio & 0.4915 & \\
\hline & $\varphi_{\mathrm{cv}}$ & $\begin{array}{l}\text { Critical state effective fric- } \\
\text { tion angle }\end{array}$ & 35.6 & \\
\hline & $c_{\mathrm{z}}$ & $\begin{array}{l}\text { Controls the strain level at } \\
\text { which fabric becomes } \\
\text { important }\end{array}$ & 200.0 & \\
\hline
\end{tabular}
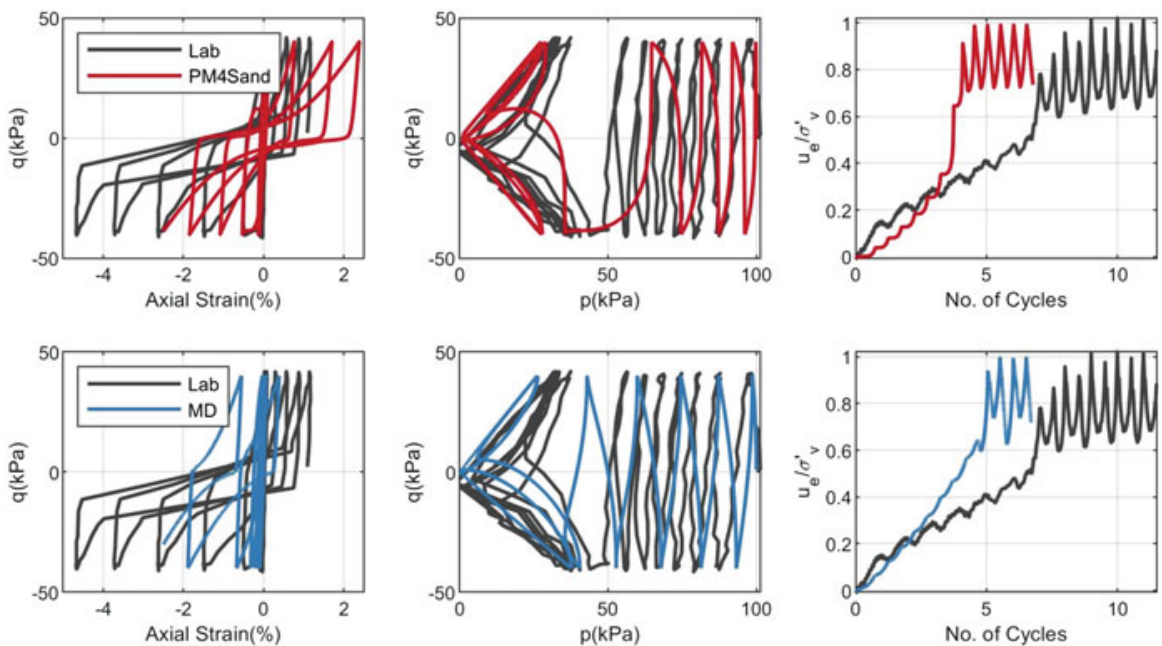

Fig. 21.4 Elemental level calibration: comparison between simulations and experimental results. The results are presented in terms of deviatoric stress, $q$, vs. axial strain; deviatoric stress, $q$, vs. mean effective stress, $p$; and excess pore pressure ratio vs. number of cycles

Fig. 21.5 Comparison of number of cycles required to reach $2.5 \%$ single-amplitude shear strain in simulations and laboratory tests

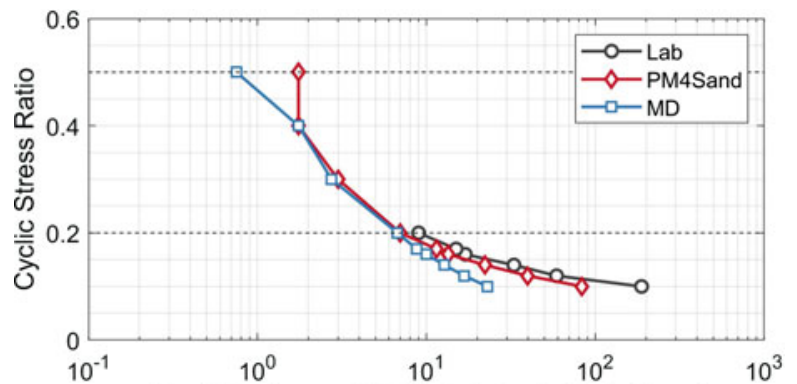

No. of Cycles until $2.5 \%$ S.A. Strain is Achieved 
test and showed that axial strains accumulate in the extension direction, while the PM4Sand model results were symmetrical in compression and extension. Both the PM4Sand and MD models underpredicted excess pore pressure during the first cycle while overpredicted overall the rate of excess pore pressure generation. Figure 21.5 depicts comparisons between simulation results for both models and laboratory test data in terms of number of cycles to initial liquefaction for different cyclic stress ratios (CSR). The number of cycles to liquefaction was evaluated counting the number of cycles necessary to reach a $2.5 \%$ single-amplitude axial strain.

\subsection{Type B Prediction Results}

By definition Type B predictions are done with limited amount of data from completed experiments. In this study, the calibrated single set of parameters for MD was used without modification. For PM4Sand, the set of parameters calibrated for the void ratio of 0.585 was chosen, and the primary parameter $D_{\mathrm{r}}$ was modified to $65 \%$. The minimum void ratio achieved in the triaxial experiments was used in all cases, while the maximum void ratio was back calculated using the achieved void ratio in each centrifuge test to match the $65 \%$ relative density. In the following subsections, results and comparisons are presented in terms of acceleration response, pore pressure response, and surface displacement.
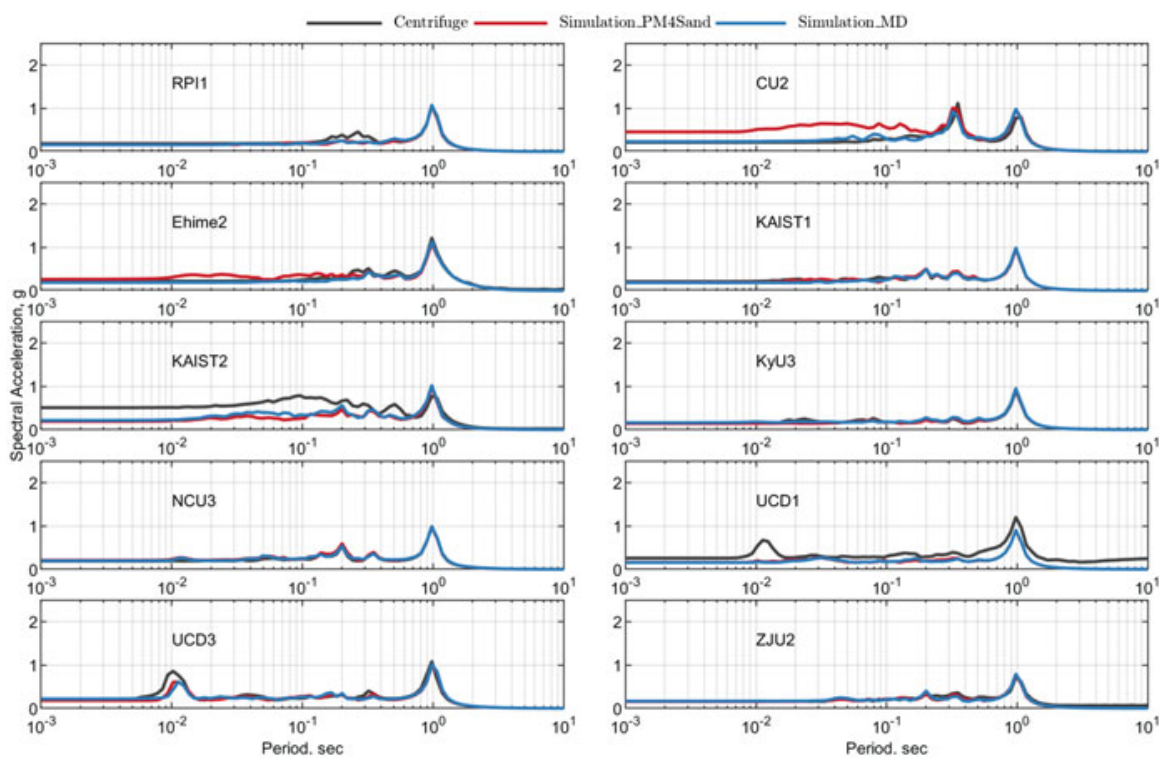

Fig. 21.6 Class-B prediction results-comparison of acceleration response spectra (5\% damping) at $\mathrm{AH} 1$ in simulations and centrifuge tests 

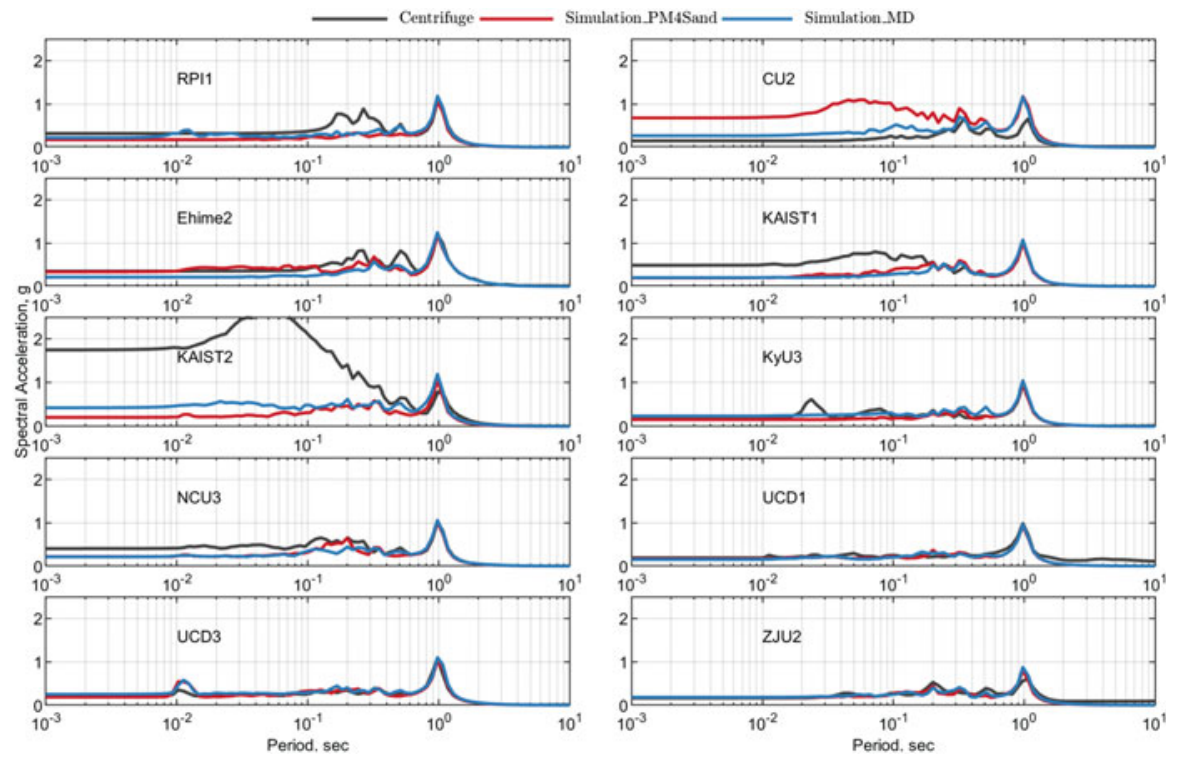

Fig. 21.7 Class-B prediction results-comparison of acceleration response spectra (5\% damping) at $\mathrm{AH} 2$ in simulations and centrifuge tests
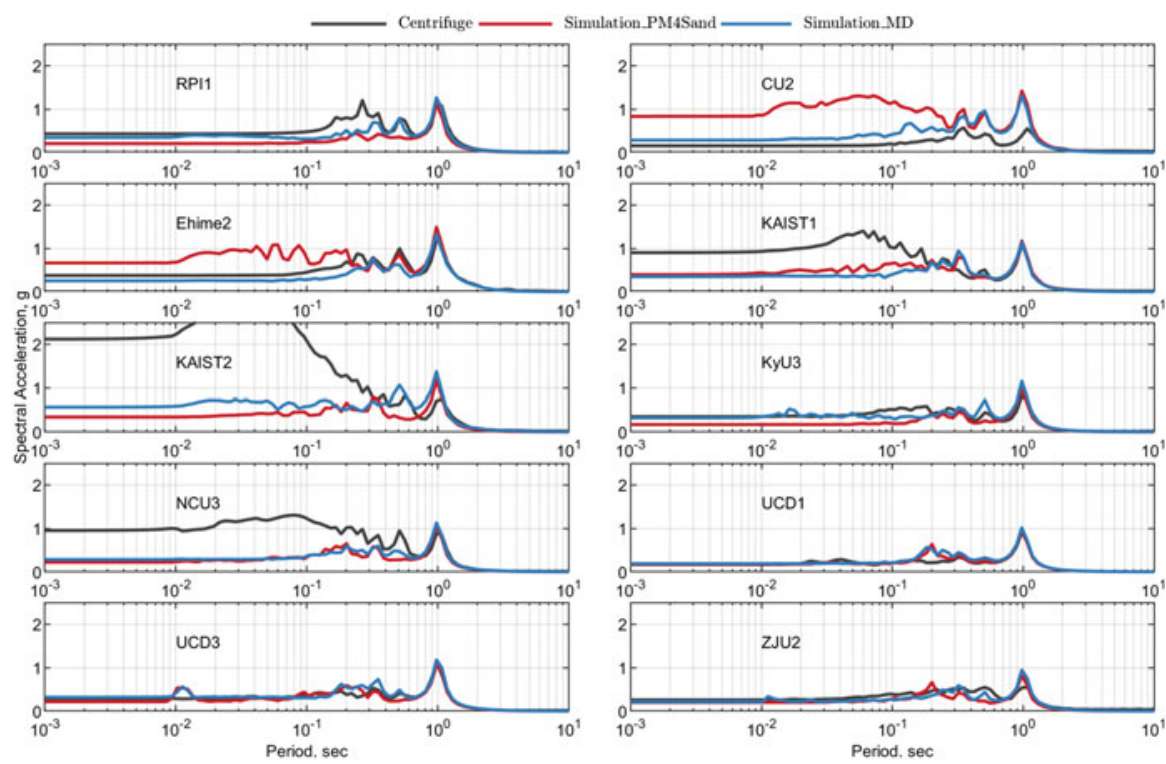

Fig. 21.8 Class-B prediction results-comparison of acceleration response spectra (5\% damping) at $\mathrm{AH} 3$ in simulations and centrifuge tests 

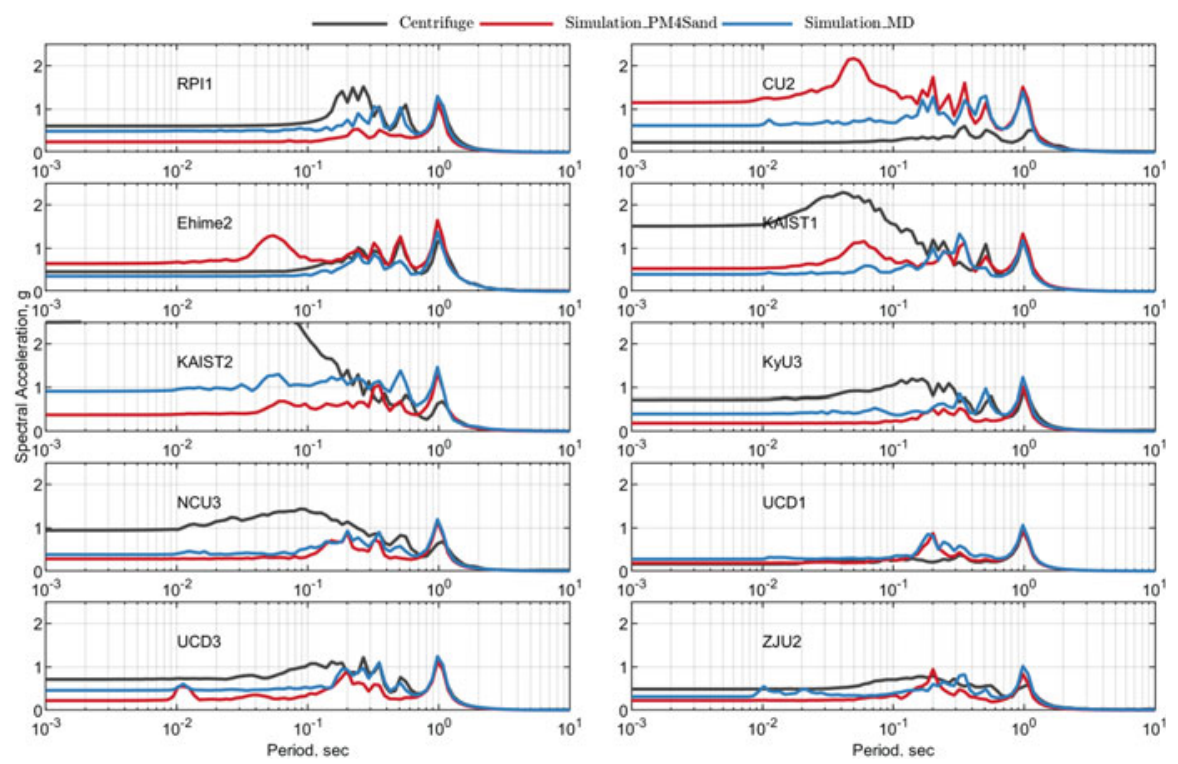

Fig. 21.9 Class-B prediction results-comparison of acceleration response spectra (5\% damping) at AH4 in simulations and centrifuge tests

\subsubsection{Acceleration Response}

Figures 21.6, 21.7, 21.8, and 21.9 show recorded and predicted acceleration response for all centrifuge experiments in terms of 5\% damping response spectra at the AH1-AH4 sensors (see Fig. 21.2 for sensor location). The plots show the simulation results predicted very well the recorded experimental data the intended $1 \mathrm{~Hz}$ input motion frequency. PM4Sand predicted higher PGAs for the CU2 and Ehime 2 experiments and generally produced results with higher frequency content. This was most likely due to material overprediction of the soil stiffness under dilation which leads to stronger simulated dilation pulses. The acceleration time histories (not shown) show even a better match.

\subsubsection{Pore Pressure Response}

All centrifuge tests showed similar trends in the pore pressure response. Comparisons of predicted and recorded pore pressure response at pore pressure sensors along centerline are depicted in Figs. 21.10, 21.11, 21.12, and 21.13. The figures show both models could capture the trend of pore pressure generation observed in the experiments. As shown in Fig. 21.5, the MD model exhibited lower cyclic resistance under lower CSRs compared to the PM4Sand model. This manifested itself in different predicted responses of pore pressure for both models. In general, MD 

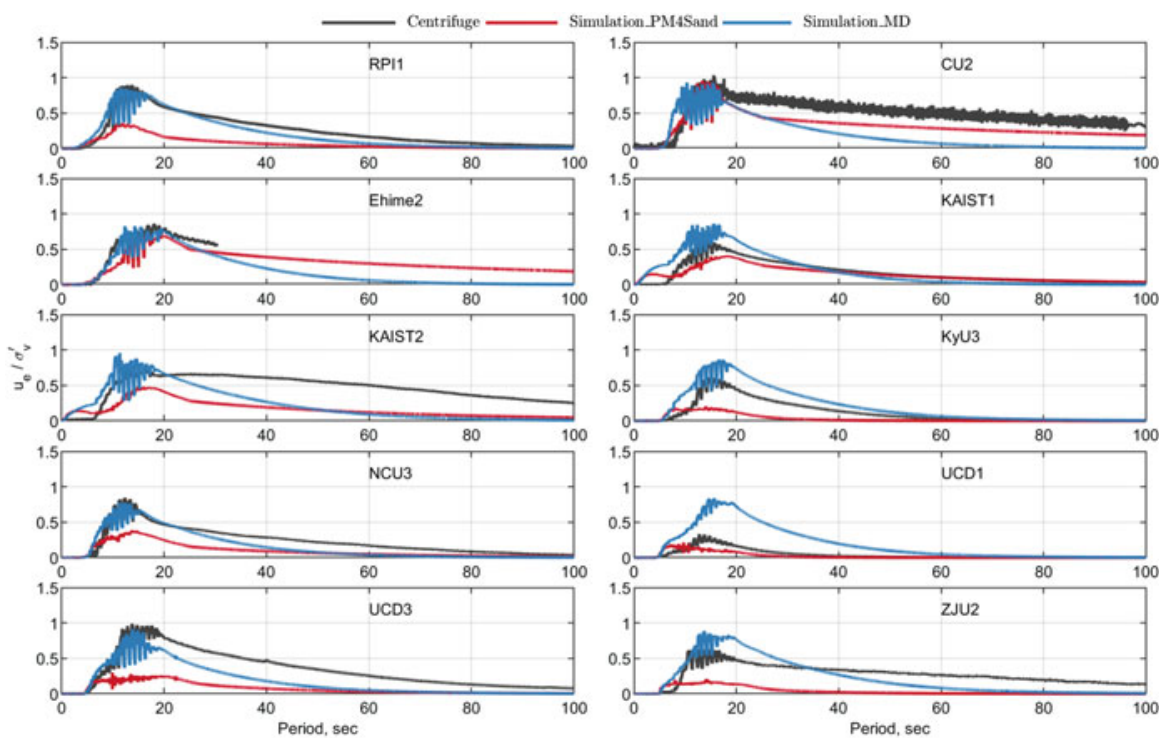

Fig. 21.10 Class-B prediction results-comparison of excess pore water pressures at P1 in simulations and centrifuge tests
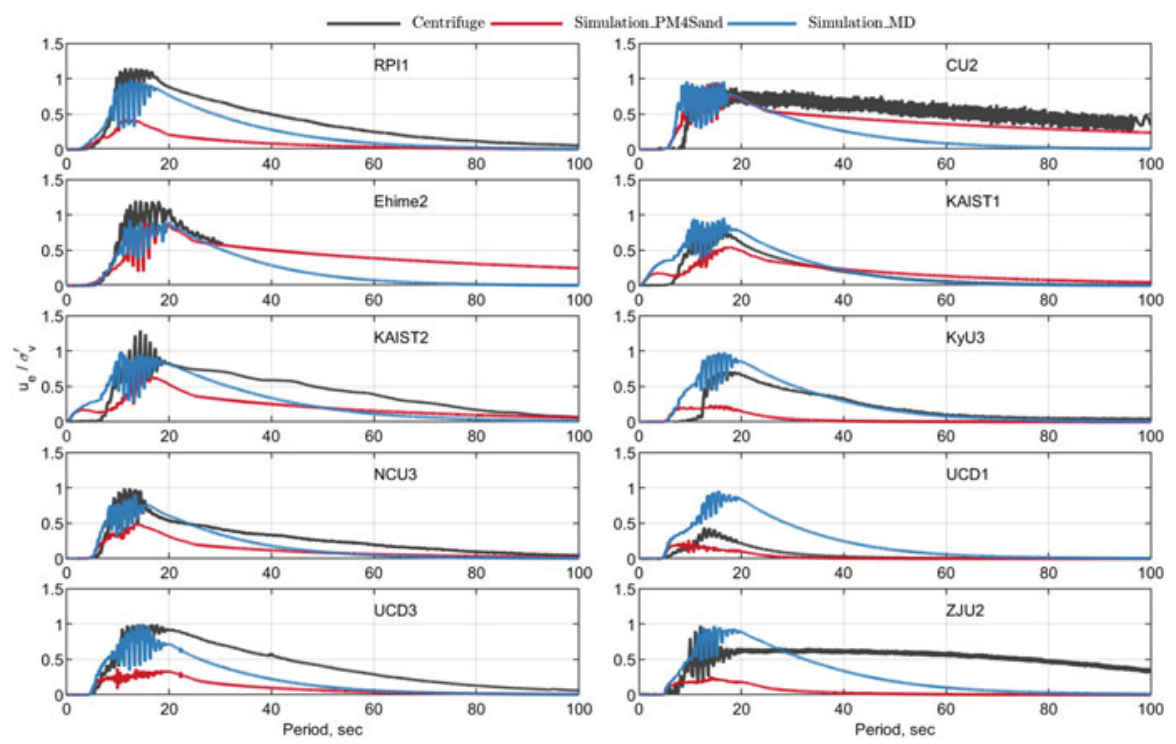

Fig. 21.11 Class-B prediction results-comparison of excess pore water pressures at $\mathrm{P} 2$ in simulations and centrifuge tests 

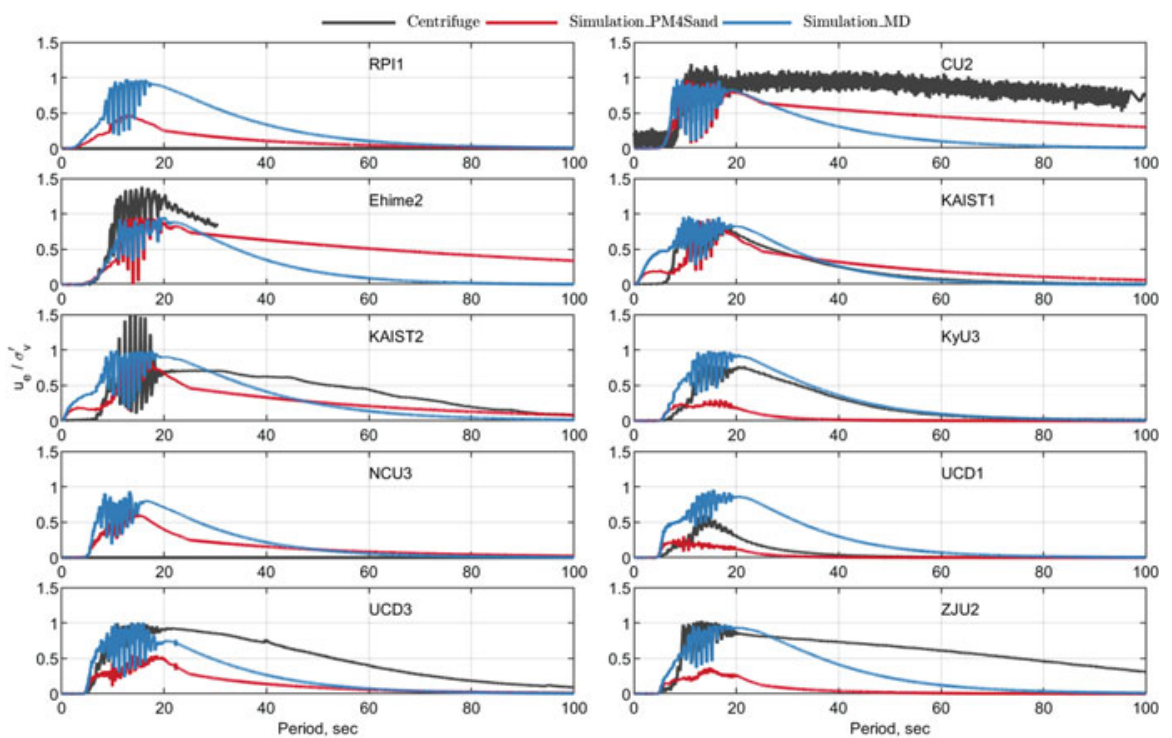

Fig. 21.12 Class-B prediction results-comparison of excess pore water pressures at P3 in simulations and centrifuge tests
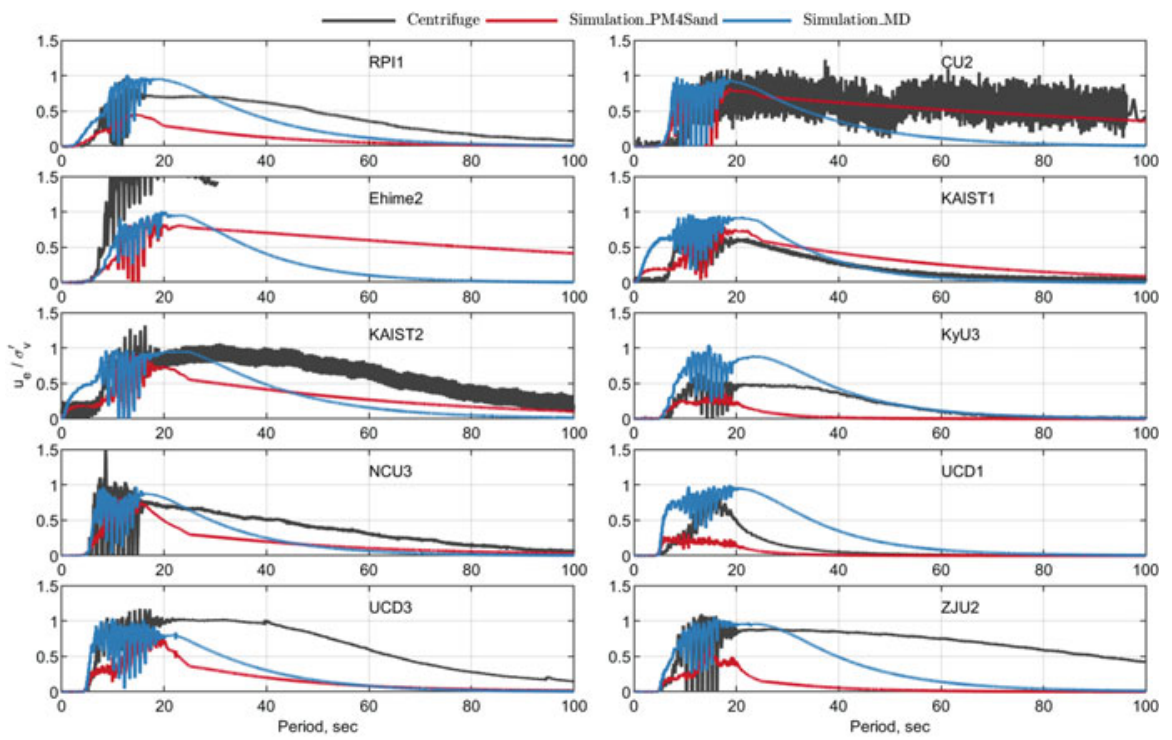

Fig. 21.13 Class-B prediction results-comparison of excess pore water pressures at $\mathrm{P} 4$ in simulations and centrifuge tests 


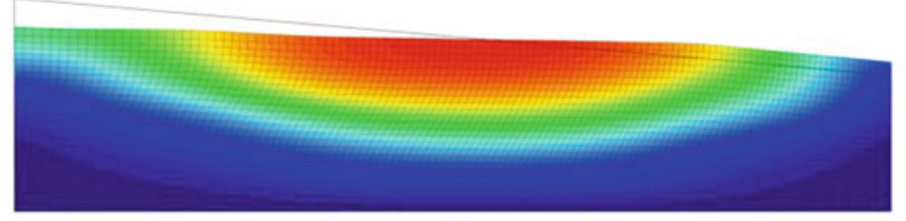

(a)
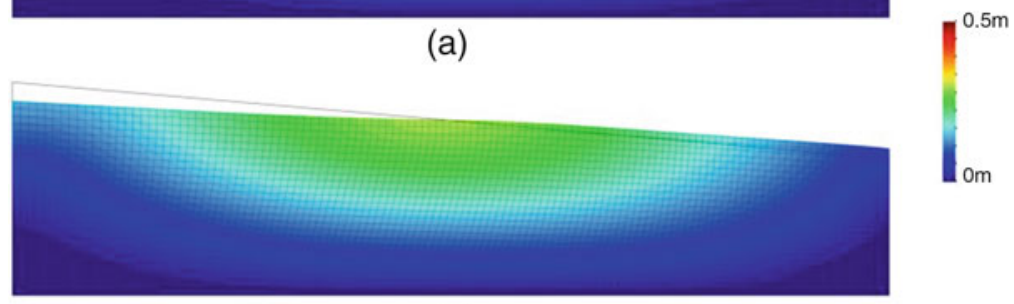

(b)

Fig. 21.14 Class-B prediction results—contour plot of absolute displacement (Ehime2). (a) PM4Sand. (b) MD

model was able to predict excess pore pressure well, while PM4Sand model underpredicted excess pore pressure generation at deeper locations. Both models showed the same excess pore pressure dissipation pattern after the main shake and until $25 \mathrm{~s}$. A slower dissipation rate of excess pore pressure shown in the simulation results after the main shake suggests that the permeability used in the numerical simulations was possibly larger than the actual value. It should be mentioned that after 25 s, the "PostShake" flag in PM4Sand model was turned "on" to achieve better post-shake settlements. Moreover, in PM4Sand the bulk modulus of the material was modified using the accumulated fabric history at each element. More details in PM4Sand can be found in Boulanger and Ziotopolou (2015).

\subsubsection{Surface Displacement Response}

Figure 21.14 depicts nodal displacements contours at the end of shaking. As expected the upslope part of the soil settled, and the downslope part heaved resulting in the flattening of the slope. Figure 21.15 depicts the evolution of recorded horizontal displacements for a surface point at the center compared to simulated results. Large variability in both centrifuge experiments and numerical simulations are observed indicating that estimation of surface displacement continues to be a challenge for both physical and numerical models. Nevertheless, despite the observed variability, both models were able to capture the evolution of displacement reasonably well. 


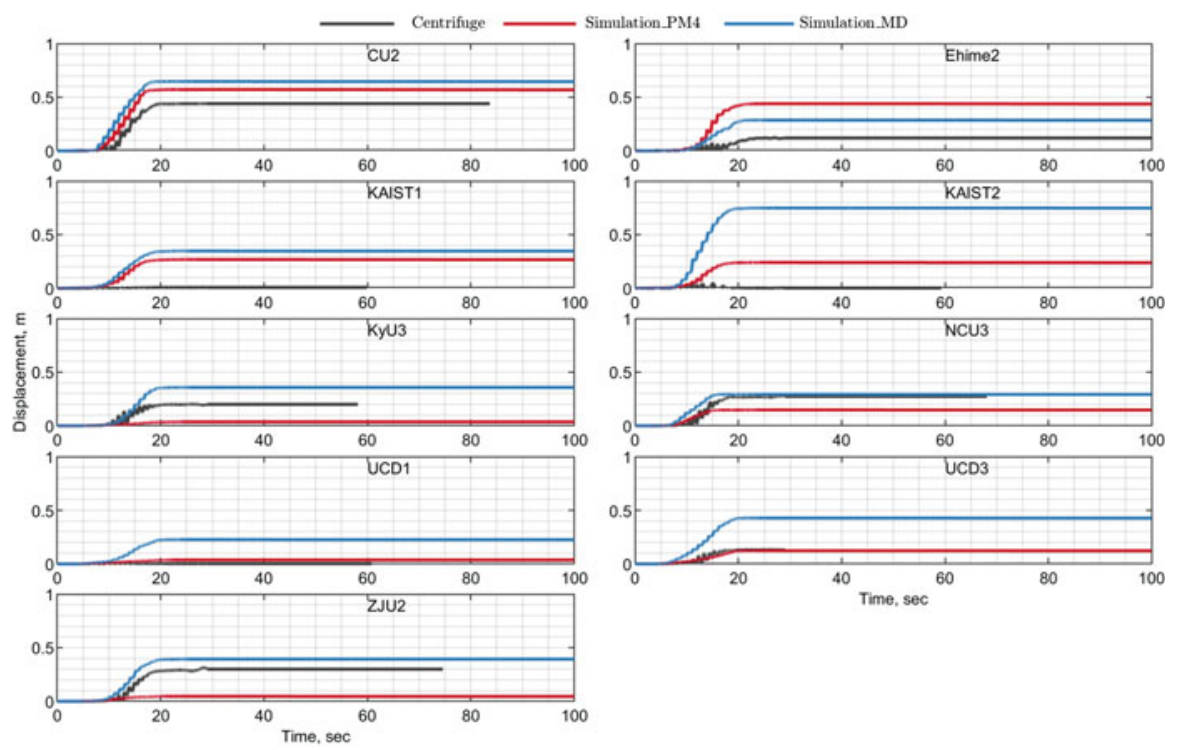

Fig. 21.15 Class-B prediction results-comparison of horizontal displacements at centerline surface point in simulation and centrifuge tests

\subsection{Conclusion}

Two constitutive models based on critical state soil mechanics and bounding surface plasticity, MD and PM4Sand, were calibrated independently to simulate the behavior of Ottawa-F65 sand in accordance with the LEAP-UCD-2017 project guidelines. The calibrated models were then used to model the response of a boundary value problem using OpenSees. Besides using different material models, the same exact finite element model was used in all simulations. Type B predictions were obtained and simulations compared to recorded data from centrifuge experiments at several facilities around the world.

The MD model was calibrated for general soil behavior, and number of loading cycles to trigger liquefaction under small CSR was not prioritized. In the calibration of the PM4Sand model parameters, the number of cycles to liquefaction observed in the laboratory test results was emphasized and used as a calibration criterion.

Type B prediction results showed that although calibrated using different methods, both models were able to predict the results from centrifuge experiments, 
especially the acceleration response at the frequency of the input motion. The evolution of excess pore pressures predicted by PM4Sand and MD were comparable and showed the similarity and differences in calibration process. The amount of predicted horizontal displacement was in the range of the recorded displacements at each facility.

\section{References}

Alawaji, H., Runesson, K., Sture, S., \& Axelsson, K. (1992). Implicit integration in soil plasticity under mixed control for drained and undrained response. International Journal for Numerical and Analytical Methods in Geomechanics, 16(10), 737-756. https://doi.org/10.1002/nag. 1610161004.

Arulanandan, K., \& Scott, R. F. (1993). Verification of numerical procedures for the analysis of soil liquefaction problems. In International Conference on the Verification of Numerical Procedures for the Analysis of Soil Liquefaction Problems (Davis, California). Rotterdam: AA Balkema.

Bastidas, A. M. P. (2016). Ottawa F-65 sand characterization. PhD thesis, University of California, Davis.

Been, K., \& Jefferies, M. G. (1985). A state parameter for sands. Géotechnique, 35(2), 99-112.

Bolton, M. D. (1986). The strength and dilatancy of sands. Géotechnique, 36(1), 65578.

Boulanger, R. W. (2003). Relating $K_{\alpha}$ to relative state parameter index. Journal of Geotechnical and Geoenvironmental Engineering, 129(8), 770-773.

Boulanger, R. W., \& Ziotopoulou, K. (2015). PM4Sand (Version 3): A sand plasticity model for earthquake engineering applications. Technical Report No. UCD/CGM-15/xx, Center for Geotechnical Modeling. Department of Civil and Environmental Engineering, University of California, Davis, CA.

Dafalias, Y. F., \& Manzari, M. T. (2004). Simple plasticity sand model accounting for fabric change effects. Journal of Engineering Mechanics, 130(6), 622-634.

El Ghoraiby, M. A., Park, H., \& Manzari, M. T. (2017). LEAP 2017: Soil characterization and element tests for Ottawa F65 sand. Washington, DC: The George Washington University.

El Ghoraiby, M. A., Park, H., \& Manzari, M. T. (2019). Physical and mechanical properties of Ottawa F65 sand. In B. Kutter et al. (Eds.), Model tests and numerical simulations of liquefaction and lateral spreading: LEAP-UCD-2017. New York: Springer.

Ghofrani, A., \& Arduino, P. (2018). Prediction of leap centrifuge test results using a pressuredependent bounding surface constitutive model. Soil Dynamics and Earthquake Engineering, 113, 758-770. https://doi.org/10.1016/j.soildyn.2016.12.001.

Kutter, B. L., Carey, T. J., Hashimoto, T., Zeghal, M., Abdoun, T., Kokkali, P., Madabhushi, G., et al. (2018). LEAP-Gwu-2015 Experiment specifications, results, and comparisons. Soil Dynamics and Earthquake Engineering, 113, 616-628. https://doi.org/10.1016/j.soildyn. 2017.05.018.

Manzari, M. T., El Ghoraiby, M., Kutter, B. L., Zeghal, M., Abdoun, T., Arduino, P., Armstrong, R. J., et al. (2018). Liquefaction experiment and analysis projects (Leap): Summary of observations from the planning phase. Soil Dynamics and Earthquake Engineering, 113, 714-743. https://doi.org/10.1016/j.soildyn.2017.05.015. 
McGann, C. R., Arduino, P., \& Mackenzie-Helnwein, P. (2015). A stabilized single-point finite element formulation for three-dimensional dynamic analysis of saturated soils. Computers and Geotechnics, 66(0), 126-141. https://doi.org/10.1016/j.compgeo.2015.01.002.

McKenna, F. T. (1997). Object-Oriented Finite Element Programming: Frameworks for Analysis, Algorithms and Parallel Computing. PhD thesis, University of California, Berkeley.

OpenSees. (2007). Open System for Earthquake Engineering Simulation. http://Opensees.berkeley. edu. University of California, Berkeley: Pacific Earthquake Engineering Research Center (PEER).

Ramirez, J., Barrero, A., Chen, L., Dashti, S., Ghofrani, A., Taiebat, M., \& Arduino, P. (2018). Site response in a layered liquefiable deposit: Evaluation of different numerical tools and methodologies with centrifuge experimental results (accepted). Journal of Geotechnical and Geoenvironmental, 144, 10.

Sloan, S. W., Abbo, A. J., \& Sheng, D. (2001). Refined explicit integration of elastoplastic models with automatic error control. Engineering Computations, 18(1/2). MCB UP Ltd), 121-194. https://doi.org/10.1108/02644400110365842.

Vasko, A. (2015). An investigation into the behavior of Ottawa sand through monotonic and cyclic shear tests. MS Thesis, The George Washington University.

Open Access This chapter is licensed under the terms of the Creative Commons Attribution 4.0 International License (http://creativecommons.org/licenses/by/4.0/), which permits use, sharing, adaptation, distribution and reproduction in any medium or format, as long as you give appropriate credit to the original author(s) and the source, provide a link to the Creative Commons license and indicate if changes were made.

The images or other third party material in this chapter are included in the chapter's Creative Commons license, unless indicated otherwise in a credit line to the material. If material is not included in the chapter's Creative Commons license and your intended use is not permitted by statutory regulation or exceeds the permitted use, you will need to obtain permission directly from the copyright holder.

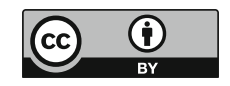

